\title{
Genome-Wide Identification of ZmHMAs and Association of Natural Variation in ZmHMA2 and ZmHMA3 with Leaf Cadmium Accumulation in Maize
}

\author{
Yanhua Cao ${ }^{1}$, Xiongwei Zhao ${ }^{2}$, Yajuan Liu ${ }^{1}$, Yalong Wang ${ }^{1}$, Wenmei Wu ${ }^{1}$, Yiwei Jiang ${ }^{3}$, Changjian Liao ${ }^{4}$, Xiaoxun \\ $\mathrm{Xu}^{5}$, Shibin Gao ${ }^{1}$, Yaou Shen ${ }^{1}$, Hai Lan ${ }^{1}$, Chaoying Zou ${ }^{1}$, Guangtang Pan ${ }^{1}$, Haijian Lin ${ }^{\text {Corresp. } 1}$ \\ ${ }^{1}$ Maize research Institute, Sichuan Agricultural University, Chengdu, Sichuan, China \\ 2 College of Life Sciences, Shanxi Agricultural University, Jinzhong, Shanxi, China \\ 3 Department of Agronomy, Purdue University, West Lafayette, Indiana, USA \\ 4 Crop Research Institute, Fujian Academy of Agricultural Sciences, Fuzhou, Fujian, China \\ ${ }^{5}$ College of Environment Sciences, Sichuan Agricultural University, Chengdu, Sichuan, China \\ Corresponding Author: Haijian Lin \\ Email address: linhj521@gmail.com
}

$\mathrm{P}_{1 \mathrm{~B}}$-type ATPases, known as heavy metal ATPases (HMAs), play an important role in the control of cadmium (Cd) accumulation in plants. In this study, a total of $12 \mathrm{ZmHMA}$ genes were identified in maize genome and particularly classified into six clusters based on their phylogenetic relationship and motif compositions. Furthermore, the expression patterns of different $Z m H M A$ genes varied with developmental stages, and were tissue specific under normal conditions. ZmHMA2 and ZmHMA3 genes exhibited significant up-regulation under $\mathrm{Cd}$ treatment. Eventually, the association analysis between 103 inbred lines and alleles in $Z m H M A 2$ and $Z m H M A 3$ revealed that one insertion-deletion (InDel) in the intron from $Z m H M A 2$ was associated with leaf $\mathrm{Cd}$ concentration under low $\mathrm{Cd}$ condition at the seedling stage. Twenty polymorphisms in ZmHMA3 were significantly associated with leaf $\mathrm{Cd}$ concentration under various $\mathrm{Cd}$ levels at seedling and maturing stages. Five single nucleotide polymorphisms (SNPs) and two InDels of these significantly associated polymorphic loci from ZmHMA3 caused the amino acid substitutions and insertion or deletion events. Importantly, the proteins encoded by ZmHMA2 and ZmHMA3 genes were located in the plasma membrane. This comprehensive analysis will provide an important theoretical basis for future functional verification of $Z m H M A$ genes to unravel the mechanisms of $\mathrm{Cd}$ accumulation in leaves of maize. Additionally, the favorable alleles in ZmHMA3 will lay a foundation for the marker-assisted selection of low Cd accumulation in maize. 
1 Genome-Wide Identification of ZmHMAs and

2 Association of Natural Variation in ZmHMA2 and

3 ZmHMA3 with Leaf Cadmium Accumulation in Maize

4 Yanhua Cao ${ }^{1, \dagger}$, Xiongwei Zhao ${ }^{2, \dagger}$, Yajuan Liu ${ }^{1}$, Yalong Wang ${ }^{1}$, Wenmei Wu ${ }^{1}$, Yiwei Jiang ${ }^{3}$,

5 Changjian $\mathrm{Liao}^{4}$, Xiaoxun $\mathrm{Xu}^{5}$, Shibin Gao ${ }^{1}$, Yaou Shen ${ }^{1}$, Hai Lan ${ }^{1}$, Chaoying Zou ${ }^{1}$, Guangtang

6 Pan $^{1}$ and Haijian Lin Corresp., 1

$8 \quad{ }^{1}$ Maize research Institute, Sichuan Agricultural University, Chengdu, China;

$9 \quad{ }^{2}$ College of Life Sciences, Shanxi Agricultural University, Jinzhong, China;

$10{ }^{3}$ Department of Agronomy, Purdue University, West Lafayette, USA;

$11{ }^{4}$ Crop Research Institute, Fujian Academy of Agricultural Sciences, Fuzhou, China;

$12{ }^{5}$ College of Environment Sciences, Sichuan Agricultural University, Chengdu, China;

$13 \uparrow$ These authors equally contributed to this work.

14 Corresponding Author: Haijian Lin

15 No. 211 Huimin road, Chengdu, Sichuan, 611130, China

16 Email address: linhj521@gmail.com

\section{Abstract}

$18 \mathrm{P}_{1 \mathrm{~B}}$-type ATPases, known as heavy metal ATPases (HMAs), play an important role in the control of cadmium $(\mathrm{Cd})$ accumulation in plants. In this study, a total of $12 \mathrm{ZmHMA}$ genes were identified in maize genome and particularly classified into six clusters based on their phylogenetic relationship and motif compositions. Furthermore, the expression patterns of different $Z m H M A$ genes varied with developmental stages, and were tissue specific under normal conditions. ZmHMA2 and ZmHMA3 genes exhibited significant up-regulation under $\mathrm{Cd}$ treatment. Eventually, the association analysis between 103 inbred lines and alleles in ZmHMA2 and $Z m H M A 3$ revealed that one insertion-deletion (InDel) in the intron from ZmHMA2 was associated with leaf $\mathrm{Cd}$ concentration under low $\mathrm{Cd}$ condition at the seedling stage. Twenty polymorphisms in $\mathrm{ZmHMA3}$ were significantly associated with leaf $\mathrm{Cd}$ concentration under various Cd levels at seedling and maturing stages. Five single nucleotide polymorphisms (SNPs) and two InDels of these significantly associated polymorphic loci from $\mathrm{ZmHMA3}$ caused the amino acid substitutions and insertion or deletion events. Importantly, the proteins encoded by ZmHMA2 and ZmHMA3 genes were located in the plasma membrane. This comprehensive analysis will provide an important theoretical basis for future functional verification of $\mathrm{ZmHMA}$ genes to unravel the mechanisms of $\mathrm{Cd}$ accumulation in leaves of maize. Additionally, the favorable alleles in $\mathrm{ZmHMA3}$ will lay a foundation for the marker-assisted selection of low $\mathrm{Cd}$ accumulation in maize. 


\section{Introduction}

Heavy metal contamination in soil has become a severe problem in many parts of the world, which threatens human life through the food chain (Li et al., 2014a). Cadmium (Cd) is one of the most toxic heavy metals present in the soil environment and is readily absorbed by vegetables and crop plants (Huang et al., 2009). Most of the Cd accumulation in the aboveground tissues, especially in the leaves of maize has become a main source of $\mathrm{Cd}$ intake in numerous developing countries as maize has been used as agricultural raw materials and as a feedstock for livestock for many years. Therefore, a better understanding of the mechanism of $\mathrm{Cd}$ accumulation in maize is an important step for breeding programs in developing varieties with low $\mathrm{Cd}$ accumulation.

$\mathrm{P}_{1 \mathrm{~B}}$-type ATPases, also named Heavy Metal ATPases (HMAs), belong to the P-type ATPase superfamily ( $\left.\mathrm{E}_{1}-\mathrm{E}_{2} \mathrm{ATPases}\right)$ that transport various metal cations across biological membranes. Through utilizing the energy generated from ATP hydrolysis, some HMA transporters act as an efflux pump to load heavy metals into the xylem from the surrounding tissues. In Arabidopsis, AtHMA2 and AtHMA4 are localized in the plasma membrane (PM) and are responsible for Cd and Zn efflux from cells (Jin et al., 2015; Verret et al., 2004). Overexpression of AtHMA4 enhances root-to-shoot translocation of Cd. In rice, OsHMA2 is also localized in the PM, and plays a role in $\mathrm{Zn}$ and $\mathrm{Cd}$ loading to the xylem and participating in root-to-shoot translocation (Satoh-Nagasawa et al., 2011; Takahashi et al., 2012b). Overexpression of OsHMA2 significantly reduces $\mathrm{Cd}$ and $\mathrm{Zn}$ accumulation in the rice leaves compared to the wild type (Takahashi et al., 2012b). However, some HMA transporters appear to assist in metal uptake and homeostasis. AtHMA3 is localized in the vacuolar membrane and its overexpression induces accumulation of $\mathrm{Cd}$ and $\mathrm{Zn}$ in the shoots and roots of Arabidopsis (Morel et al., 2009). On the contrary, OsHMA3, also localized in the vacuolar membrane, limits Cd translocation from the roots to the aboveground tissues (Ueno et al., 2010). Given the diverse biological function of different $H M A$ genes, their roles in $\mathrm{Cd}$ accumulation needs to be further explored in plant species.

Although some $H M A$ genes have been functionally characterized in Arabidopsis and rice, the detailed characterization and functions of $H M A$ family members for $\mathrm{Cd}$ translocation remain unknown in maize. In the present study, we investigated phylogenetic relationship, gene structure, expression pattern, and motif composition of $H M A$ family genes in maize. In addition, association analysis between the genetic variations of ZmHMA2 and ZmHMA3 and plant response to $\mathrm{Cd}$ stress was conducted in a diverse maize population consisting of 103 inbred lines from tropical and temperate regions. The objective of the study was to identify specific ZmHMA genes that control $\mathrm{Cd}$ transport and to analyze the loci causing functional variation of the target genes. In addition, the favorable alleles derived from polymorphic loci within ZmHMA2 and 
$71 \mathrm{ZmHMA3}$ will provide the foundation for the marker-assisted selection of low Cd accumulation 72 in maize breeding. 


\section{Materials and Methods}

74

75

76

77

78

79

80

81

82

83

84

85

86

87

88

89

90

\section{Plant Materials and Phenotyping}

Two experiments were conducted for analyzing $\mathrm{Cd}$ accumulation in leaves of 269 inbred lines. Detailed information of experimental design and plant growing conditions were described previously (Zhao et al., 2018). Briefly, for a controlled environment study, maize seedlings of 269 inbred lines were planted in plastic pots containing $14 \mathrm{~kg}$ clayey soil under natural sunlight in a greenhouse in 2015 and 2016, respectively. In order to simulate the extent of Cd pollution of urban soil in China (Li et al., 2014b), the middle-Cd treatment were applied as $\mathrm{CdCl}_{2} \cdot 2.5 \mathrm{H}_{2} \mathrm{O}$ at $0.1 \mathrm{mmol} \cdot \mathrm{kg}^{-1}$ (available $\mathrm{Cd} 18.8 \mathrm{mg} \cdot \mathrm{kg}^{-1}$ in soil), while no $\mathrm{CdCl}_{2} \cdot 2.5 \mathrm{H}_{2} \mathrm{O}$ was added to the soil for low-Cd treatment (available Cd of $3.28 \mathrm{mg} \cdot \mathrm{kg}^{-1}$ in soil). Seedlings ( $18 \mathrm{~d}$ old) were cultured under low-Cd condition and middle-Cd conditions in a randomized complete block design with two replicates. After 15 days, the third and fourth leaves were harvested to analyze $\mathrm{Cd}$ accumulation in maize leaves. The traits for the pot experiment at seedling stage were labelled LCd15-Low (Leaf Cd concentration under low-Cd level in 2015), LCd16-Low (Leaf Cd concentration under low-Cd level in 2016), LCd15-Middle (Leaf Cd concentration under middleCd level in 2015), and LCd16-Middle (Leaf Cd concentration under middle-Cd level in 2016).

For the field experiment, the inbred lines were planted in a highly Cd-contaminated soil $(32.5 \mathrm{Cd}$ $\mathrm{mg} \cdot \mathrm{kg}^{-1}$ soil) in Deyang city in 2015 and 2016. The field experiment was a randomized complete block design with two replications. After the seeds matured, five consecutive plants were chosen from the middle of each row, and the middle leaf below the tassel was harvested for measuring Cd concentration (LCd15-Field and LCd16-Field).

Harvested leaf samples were rinsed with tap water, washed three times with deionized water, and then dried at $70^{\circ} \mathrm{C}$ in an oven until completely dried. The dry samples were ground to a powder using a pestle with liquid nitrogen. Subsequently, the $\mathrm{Cd}$ concentration was determined by using inductively coupled plasma-atomic emission spectrometry (ICP-MS) as described previously.

\section{Identification of HMA Family in Maize and Phylogenetic Analysis}

To identify the $H M A$ genes in maize, the known amino acid sequences of the published cloned OsHMA2 and OsHMA3 (Takahashi et al., 2012a; Ueno et al., 2010) were used in the BLASTP program against the maize B73 reference genome (B73 RefGen_v3, https://www.maizegdb.org/). The protein was selected as a candidate protein only when passing the expected threshold of 1e-10, and amino acid sequence $>200$ residues. Each annotated protein was examined for the existence of E1-E2 ATPase domain (PF00122) by SMART (http://smart.embl-heidelberg.de/). Multiple sequence alignment was performed by the 
107 ClustalW2.1 program with default parameters (Larkin et al., 2007). Transmembrane domains

108 were predicted with internet-programs SOSUI (http://bp.nuap.nagoya-u.ac.jp/sosui/).

109 The phylogenetic analysis among maize, rice and Arabidopsis was conducted with the

110 neighbor joining (NJ) method (1,000 bootstrap replicates) using ClustalW2.1 based on the full-

111 length protein alignment. The phylogenetic tree was displayed in FigTree software. The exon-

112 intron structure of ZmHMA genes was graphically displayed by the Gene Structure Display

113 Server (Hu et al., 2014). The ZmHMA protein sequences were used to predict the conserved

114 motifs by using the MEME Suite web server (http://meme-suite.org/) (Bailey et al., 2009) with

115 the maximum number of motif sets at 10 and the optimum width of motifs from 5 to 300 amino

116 acids.

\section{Gene Expression Analysis}

118 Expression patterns of $Z m H M A$ genes in different maize tissues were analyzed using the

119 genome-wide gene expression atlas of maize inbred B73 line that was reported previously

120 (Stelpflug et al., 2016). Expression data under normal condition for the 6 tissues were combined

121 from 79 distinct replicated samples (RNAseq) (Supplementary Table S1). In addition, the

122 responsiveness of each $Z m H M A$ genes to $\mathrm{Cd}$ stress was analyzed by quantitative real-time PCR

123 (qRT-PCR). Briefly, the 2-week-old plants of the B73 (a line with low Cd accumulation and a

124 reference genome of maize) were grown in $1 / 2$ Hoagland's nutrient solution amended with

$125 \mathrm{CdCl}_{2} \cdot 2.5 \mathrm{H}_{2} \mathrm{O}\left(200 \mu \mathrm{mol} \cdot \mathrm{L}^{-1}\right)$ for $0 \mathrm{~h}$ (used as control), $12 \mathrm{~h}, 24 \mathrm{~h}$, and $48 \mathrm{~h}$. Total RNA from the

126 roots, stems and leaves was isolated using TRIZOL reagent (Invitrogen, USA) and then reverse

127 transcription was performed with PrimeScript RT Reagent Kit (Takara, Japan). The primers used

128 in the qRT-PCR experiments were designed by Primer 5.0 software and listed in Supplementary

129 Table S2. GADPH was used as a housekeeping gene for normalization in the present study.

130 Subsequently, qRT-PCR was conducted using the SYBR premix Ex Taq kit (Takara) on an ABI

1317500 Real-Time System (Applied Biosystems) as follows: $95^{\circ} \mathrm{C}$ for $30 \mathrm{~s} ; 95^{\circ} \mathrm{C}$ for $5 \mathrm{~s}, 60{ }^{\circ} \mathrm{C}$ for

$13230 \mathrm{~s}, 40$ amplification cycles, and then the melt curves were generated for verification of

133 amplification specificity by a thermal denaturing step. The method of $2^{-\Delta \Delta C T}$ was used to

134 calculate the relative gene expression level between times (Schefe et al., 2006). The analysis

135 included three biological replicates and three technical replicates for each sampling time.

$136 \mathbf{Z} \boldsymbol{m H M A 2}$ and $\mathbf{Z} \boldsymbol{m H M A 3}$ Gene Sequences and Association Analysis with Cd Accumulation

137 in Maize

138 In total, 103 maize inbred lines (stiff stalk, non-stiff stalk, and tropical or subtropical group) were

139 randomly selected from a natural maize population of 269 inbred lines (Supplementary Table S3),

140 and used for an association analysis. To identify the sequence variations among candidate genes

Peer) reviewing PDF | (2019:04:37193:2:0:NEW 30 Aug 2019) 
141 associated with Cd accumulation the ZmHMA2 (GRMZM2G099191) and ZmHMA3

142 (GRMZM2G175576), full sequences (5'-UTR, exons, intron and 3'-UTR) of these two genes

143 were amplified from genomic DNA in 103 maize inbred lines using high-fidelity polymerase

144 KOD FX Neo (TaKaRa). Three pairs of primers were designed using the B73 genome sequence

145 as a reference and Primer 5.0 software (Supplementary Table S2). A 150 bp overlap between

146 each pair of primers was designed in the target regions. PCR products from 103 inbred lines

147 were purified using E.Z.N.A and sequenced directly using the ABI 3730 sequencer. These

148 sequences were aligned using MUSCLE 3.8, assembled using ContigExpress, and manually

149 corrected using BioEdit 7.1 software (Edgar 2004).

$150 \quad$ Nucleotide polymorphism in ZmHMA2 and ZmHMA3, including SNP and InDels, was

151 identified and extracted (MAF $\geq 0.05)$. Allelic diversities with parameters of nucleotide diversity

$152(\pi)$ and nucleotide polymorphism $(\theta)$ were calculated using DnaSP 6.0 software (Rozas et al.,

153 2017). Tajima's $D$ statistics and Fu and Li's statistical tests of ZmHMA2 and ZmHMA3 were

154 selected to investigate the evolutionary pressure within the different regions. The significance of

155 each DNA polymorphism associated with Cd leaf concentration of maize was calculated using a

156 mixed linear model (MLM) implementing both population structure (Q) and kinship (K) in

157 TASSEL 3.0 (Bradbury et al., 2007; Liu et al., 2015). The Q and K were calculated using

158 genome wide SNPs as described by Zhao et al (2018). The significance threshold of $P<0.01$

159 was used for the candidate gene-based association analysis. Levels of linkage disequilibrium

160 (LD) between pairs of two polymorphic loci were estimated using Haploview v4.2 (Barrett et

161 al., 2004).

\section{Subcellular Localization of ZmHMA2 and ZmHMA3 Proteins}

163 We used ProtComp version 9 (http://linux1.softberry.com/berry.phtml) for comparing

164 homologous proteins of known localization and pentamer distributions in the LocDB and

165 PotLocDB databases to predict the putative protein subcellular localization. For verifying the

166 prediction, the coding regions of $Z m H M A 2$ and $Z m H M A 3$ without the stop codon were amplified

167 from B73 cDNA and cloned downstream of the 35S promoter in the pCAMBIA2300 vector that

168 carried GFP. The primers used to amplify ZmHMA2 and ZmHMA3 were listed in Supplementary

169 Table S2. The PCR products were digested with BamHI, and directionally ligated into vector

170 pCAMBIA2300 to construct the ZmHMA2-GFP or ZmHMA3-GFP fusion gene driven by a 35S

171 promoter (Liu et al., 2010), respectively. In addition, equal volume suspensions of

172 Agrobacterium strain GV3101 harboring Ti plasmids expressing either ZmHMA2-GFP and a

173 plasma marker (PIP2A-RFP) or ZmHMA3-GFP and a plasma marker (PIP2A-RFP) were mixed

174 (final $O D 600=0.6$ ). These plasmids were individually expressed or co-expressed in tobacco 
175 (Nicotiana benthamiana) leaves by Agrobacterium-mediated infiltration. GFP and RFP signals

176 were visualized using a fluorescence confocal scanning microscope (Nikon A1 i90, LSCM,

177 Japan) at $72 \mathrm{~h}$ after infiltration.

\section{Results}

\section{Identification and Properties of Putative Maize $\boldsymbol{H} \boldsymbol{A}$ Genes}

$180 \mathrm{P}_{1 \mathrm{~B}}$-type ATPases (HMAs) in maize were identified using the cloned OsHMA2 (Oryza sativa

181 Japonica) and OsHMA3 (Oryza sativa Japonica) as protein queries in BLASTP searches against

182 the B73 reference genome sequence. A total of $12 \mathrm{HMA}$ genes were identified and were named

$183 \mathrm{ZmHMA1}$ to ZmHMA12 (Table 1) following the nomenclature of rice. These ZmHMA genes

184 were unevenly distributed along the 5 out of 10 maize chromosomes. Four ZmHMAs were

185 present on chromosome 2; three on chromosome 5; and only one gene each on chromosomes 1

186 and 9. The relatively high densities of $\mathrm{ZmHMA}$ genes were observed at the central sections of

187 chromosomes including the centromeres and the pericentromeric regions (Table 1). Only

188 ZmHMA6 and ZmHMA11 were noted at the bottom of chromosome 4. Gene structure analysis

189 revealed that most $Z m H M A$ genes displayed the complex exon-intron structure. Seven ZmHMA

190 genes have more than nine exons. The predicted molecular weights of the 12 deduced ZmHMA

191 proteins ranged from 42.3 (ZmHMA12) to $112.43 \mathrm{kDa}$ (ZmHMA2) (Table 1). These results

192 indicated that the $H M A$ genes were highly conserved over the course of evolution since the

193 pericentromeric regions of chromosomes have reduced recombination rates.

\section{Phylogenetic and Genomic Structure Analysis of Maize HMA Proteins}

195 To investigate the phylogenetic relationship between the $H M A$ gene family in maize,

196 Arabidopsis and rice, the amino acid sequences of 12 HMA members from maize, 8 from

197 Arabidopsis and 9 from Oryza sativa were used to construct a phylogenetic tree. Based on the

198 phylogenetic tree, it clearly showed that all the maize HMA proteins were classified into the

199 same corresponding categories in Arabidopsis and O. sativa, which include HMA I, HMA II,

200 HMAIII, HMAIV, HMA V and HMAVI clusters (Figure 1). Functional annotation against gene

201 ontology terms (GO; www.geneontology.org) shown that all ZmHMAs were involved in

202 ATPase-coupled transmembrane transporter activity, cation transport and ATP binding

203 (Supplementary Table S4). ZmHMA1, ZmHMA1, ZmHMA3 and ZmHMA4 (HMA I, HMA II )

204 were involved in Cadmium/Zinc ion transmembrane transport (GO:0070574, GO:0071577) for

205 biological process categories. Eight of these ZmHMAs (HMAIII-HMAVI) were listed as 
206 involved in Copper $(\mathrm{Cu})$ ion binding (GO:0005507) for molecular function. In addition, each

207 cluster contained at least one Arabidopsis and rice member in the HMA clusters (Figure 1,

208 Figure 2A), which suggests that these homologous genes may have derived from multiple

209 duplications during the evolution.

210 Due to the different lengths and abundant diversity of the functional domains of the HMA

211 proteins, domain features were used for diversity analyses. As shown in Supplementary Figure

$212 \mathrm{~S} 1$, a total of 10 conserved motifs were identified in ZmHMA proteins by the protein domain

213 analysis, including motifs 10 and 8 encoding the heavy-metal-associated domain (HMA

214 domain), motifs 3, 5, 2 encoding E1-E2 ATPase domain, and motifs 4, 1 encoding the hydrolase

215 domain. As expected, all the ZmHMA proteins possess the typical structures of the $\mathrm{P}_{1 \mathrm{~B}}$-type

216 ATPases, including a E1-E2 ATPase domains (PF00122) (Figure 2B). However, there were still

217 obvious differences in the domain composition among these clusters. For instance, clusters $\mathrm{V}$

218 and VI had the largest number of motifs, while there only two motifs in cluster I . The HMA

219 domain was absent in ZmHMA1 and ZmHMA12. ZmHMA1, ZmHMA2 and ZmHMA8 lacked

220 the hydrolase domain (Figure 2B). Analysis of transmembrane helices suggested that the proteins

221 of cluster I had 6 to 9 predicted transmembrane domains (TMs) (Supplementary Figure S2,

222 Supplementary Table S5). Notably, ZmHMA3 and ZmHMA4 had exact similarity of gene

223 structure on the same chromosome (Figure 2C).

224 Expression Analyses of $\mathbf{Z} \boldsymbol{m H M A}$ Genes

225 To investigate the expression patterns of $Z m H M A$ genes in different tissues, an expression

226 heatmap was constructed for 12 ZmHMA genes in the 13 different tissues of the B73 under

227 normal conditions (Figure 3A). The results indicated that the expression patterns of different

$228 Z m H M A$ genes varied greatly among tissues. Of them, the transcripts of ZmHMA2, ZmHMA6,

$229 \mathrm{ZmHMA7,ZmHMA11}$ and ZmHMA12 were highly and constitutively expressed in the various

230 tissues. The $Z m H M A 3$ transcript was expressed at a relatively high level in roots and nodes

231 compared to other tissues. On the contrary, ZmHMA1 and ZmHMA4 were not expressed or

232 exhibited at extremely low level in different tissues (Figure 3A).

233 To determine which ZmHMA genes responded to Cd stress, the expression level of 12

234 ZmHMA genes was analyzed in B73 roots, stems, leaves under the Cd treatment using qRT-PCR.

$235 \mathrm{ZmHMA4}$ failed to be amplified in roots, leaves and stems. We found that the expression levels

236 of ZmHMA1, ZmHMA3, ZmHMA5 and ZmHMA12 in the roots were significantly upregulated

237 by the Cd treatment (Figure 3B, Figure 3D, Figure 3E, Figure 3L). Among these genes, 
238 ZmHMA3 was highly induced in roots and was upregulated more than 21 -fold after $48 \mathrm{~h}$ of $\mathrm{Cd}$

239

240

241

242

243

244

245

246

247

248

249

250

251

252

253

254

255

256

257

258

259

260

261

262

263

264

265

266

267

268

269

270

271

treatment compared to normal growing conditions. In leaves, the expression levels of ZmHMA2, ZmHMA3, ZmHMA7 and ZmHMA12 were significantly induced by Cd treatment (Figure 3B, Figure 3C, Figure 3G, Figure 3L). Specifically, the remarkably higher expression of ZmHMA2 was found in leaves with a 15 -fold increase after $12 \mathrm{~h}$ of $\mathrm{Cd}$ treatment. The other nine ZmHMA genes, ZmHMA1, ZmHMA2, ZmHMA5, ZmHMA6, ZmHMA7, ZmHMA8, ZmHMA9, ZmHMA10 and $Z m H M A 11$, were markedly downregulated in the stems. Collectively, the data indicated that different $Z m H M A$ genes exhibited variable levels of expression in different tissues and developmental stages of maize.

\section{ZmHMA2 and ZmHMA3 Gene Polymorphisms and LD Decay}

Previous functional research reported that OsHMA1-OsHMA3 (Oryza sativa Japonica) belonged to zinc $(\mathrm{Zn}) /$ cobalt $(\mathrm{Co}) /$ cadmium $(\mathrm{Cd}) /$ lead $(\mathrm{Pb})$ transport (Takahashi et al., 2012a). OsHMA2 plays an important role in root-to shoot translocation of $\mathrm{Zn}$ and $\mathrm{Cd}$, and OsHMA3 is important for limiting the $\mathrm{Cd}$ in above-ground tissues (Takahashi et al., 2012b; Ueno et al., 2010). On the basis of phylogenetic analysis, the ZmHMA2 protein exhibited 70\% identity with OsHMA2 and ZmHMA3, and 72\% with OsHMA3. Comprehensive analysis highlighted ZmHMA2 and $\mathrm{ZmHMA3}$, as candidate genes of particular interest.

To determine whether ZmHMA2 and ZmHMA3 genes were associated with Cd accumulation in leaves of maize, the natural variations within $Z m H M A 2$ and $Z m H M A 3$ genes were detected by determining the levels of nucleotide diversity. Sequence polymorphisms were detected among 103 maize inbred lines across 6,626 bp of ZmHMA2 and $3966 \mathrm{bp}$ of $\mathrm{ZmHMA3}$, which covered 5'-UTR, exons, introns and 3'-UTR. SNPs and InDels at the ZmHMA2 and ZmHMA3 locus were identified (Supplementary Table S6, Supplementary Table S7). From the putative genomic sequences in the 103 inbred lines, 205 SNPs and 62 InDels were detected from ZmHMA2, with one SNP and InDel every 23 and103 bp, respectively (MAF $\geq 0.05$ ) (Supplementary Table S6). In addition, 139 SNPs and 24 InDels events were found in ZmHMA3, with one SNP and one InDel every 27 and 157 bp, respectively (Supplementary Table S7). Nucleotide diversity analysis using sliding windows indicated that introns of $Z m H M A 2$ had the highest nucleotide diversity $(\pi$ $\left.=17.58 \times 10^{-3}\right)$ and its exon had the lowest nucleotide diversity $\left(\pi=6.24 \times 10^{-3}\right)$ in maize (Table 2). However, in ZmHMA3, the estimates of nucleotide diversity in the 3'-UTR $\left(\pi=33.57 \times 10^{-3}\right)$ was obviously higher than that of other regions. Fu and Li's $F$ and $D$ test of each region showed that the exon of ZmHMA2 and the 3'UTR of ZmHMA3 were under purifying selection with significantly positive values. These results suggested that purifying selection and/or population size expansion had occurred in the two regions respectively. In addition, the combined LD

Peer] reviewing PDF | (2019:04:37193:2:0:NEW 30 Aug 2019) 
272 analysis of ZmHMA2 and ZmHMA3 showed LD decay was close to 0.1 within $1300 \mathrm{bp}$ (Figure 273 4).

$274 \boldsymbol{Z m H M A 2}$ and $\mathrm{ZmHMA3}$ Genes Associated with Cd Accumulation in Leaves of Maize

275 The association analysis between natural variations in $Z m H M A 2$ and $Z m H M A 3$ and $\mathrm{Cd}$

276 concentration in maize were performed using the $\mathrm{Q}+\mathrm{K}$ model. One co-located InDel in the intron

277 (InDel S1174, $P=7.96 \times 10^{-3}$ ) in $Z m H M A 2$ was associated with leaf $\mathrm{Cd}$ concentration at seedling

278 stage under low Cd condition in 2015 and 2016 (LCd15-Low and LCd16-Low) (Table 3). For

279 ZmHMA3, 20 significant loci (15 SNPs and 5 InDels) were associated with leaf Cd concentration

280 under various soil environments at seedling and maturing stages in 2015 and $2016(P<0.01)$

281 (Figure 5, Table 3). $\mathrm{R}^{2}$ marker values ranged from 7.7 to $16.3 \%$ among all those associated loci.

282 On average, each site explained $11.2 \%( \pm 3.3 \%)$ of phenotypic variance for its associated trait.

283 Among the 20 loci, S917 located on the second exon region were significantly associated with

284 LCd16-Middle $(P<0.01)$ (Table 3). Moreover, eight loci (S3097, S3114, S3297, InDel S3517,

285 InDel S3594, S3692, S3695, S3792 ) with a complete LD were significantly associated with

286 LCd15-Field and LCd16-Field. Furthermore, 5 SNPs and 2 InDels were located on the CDS

287 region and caused the amino acid substitutions and insertions or deletions.

288 Putative $\boldsymbol{Z} \boldsymbol{m} \boldsymbol{H} \boldsymbol{M} \boldsymbol{A} 2$ and $\boldsymbol{Z} \boldsymbol{m H} \boldsymbol{H} \boldsymbol{A} 3$ Effect on Cd Accumulation

289 Based on the average phenotype of plants grown in 2015 and 2016, the effects of gene

290 polymorphisms on $\mathrm{Cd}$ accumulation were further analyzed using mixed linear model. Genotype

291 with a deletion of GCA (InDel S1174) in an intron of ZmHMA2 exhibited a significantly higher

292 Cd concentration than that of the genotype with an insertion of GCA (InDel S1174) under low

$293 \mathrm{Cd}$ condition at seedling stage (Figure 6A). The individuals carrying the minor frequency alleles

294 had a $1.03 \mathrm{mg}$ lower leaf $\mathrm{Cd}$ content than those with major frequency alleles $\left(P=8.48 \times 10^{-3}\right)$. In

295 addition, the site S917 (CC) associated with LCd16-Middle at seedling stage had one amino acid

296 substitution from alanine (A) to glycine (G) $\left(P<2.09 \times 10^{-3}\right)$. The Cd concentration of leaves in

297 the genotype at seedling stage with SNP G/G was about 6.9 mg higher than those with SNP CC

298 (Figure 6B). In the field study across 2015 and 2016, four polymorphic loci (S3114, S3297,

299 InDel S3517, InDel S3594) in the CDS region of ZmHMA3 were significantly co-associated with

300 LCd16-Field and LCd15-Field. From the two SNP positions, two amino acid substitutions, from

301 glycine (G) to alanine (A) at residue 3114 and from valine (V) to alanine (A) at residue 3296,

302 were identified (Table 3). More importantly, InDel S3517 (GTCCCGTGA) encoded valine (V),

303 proline (P) and the stop codon. In addition, InDel S3517 and the three loci above showed a

304 complete LD pattern. A significant difference in $\mathrm{Cd}$ accumulation of leaves among the

305 accessions with homozygous allelic SNP S3114, S3297 and InDel S3517 in ZmHMA3 was found 
306 at mature stage in the field study (Figure 6C). The inbred lines with major frequency alleles

307 (GG+TT+9) exhibited a significantly lower $\mathrm{Cd}$ accumulation in leaves than that of inbred lines

308 with minor frequency alleles $(\mathrm{CC}+\mathrm{CC}+0)\left(P<2.82 \times 10^{-3}\right)$. The $\mathrm{Cd}$ accumulation in mature

309 leaves of individuals with minor frequency alleles was significantly reduced by $10.96 \mathrm{mg}$

310 compared to those with major frequency alleles.

\section{Subcellular Localization of ZmHMA2 and ZmHMA3 Proteins}

312 Possible subcellular locations of ZmHMA2 and ZmHMA3 proteins were predicted using

313 ProtComp 9.0. This prediction suggested that ZmHMA2 and ZmHMA3 might be localized on

314 PM (Supplementary Table S8). To examine the predictions above, the control vector

$315 p C A M B I A 2300-35 S:: G F P$, the loaded vector $p C A M B I A 2300-35 S:: Z m H M A 2-G F P$ and

$316 p C A M B I A 2300-35 S:: Z m H M A 3-G F P$, were transferred into tobacco using transient

317 transformation. ZmHMA2 and ZmHMA3 proteins were successfully expressed as fluorescent

318 protein fusions (ZmHMA2-GFP and ZmHMA3-GFP) (Figure 7). Compared with the control

319 (Figure 7D), the green fluorescence signals of ZmHMA2-GFP (Figure 7E) and ZmHMA3-GFP

320 (Figure 7F) were preferentially detected in the cytoplasm of the tobacco leaf epidermis. To

321 further confirm subcellular localization of proteins, we co-infiltrated the tobacco leaves with

322 Agrobacterium containing the PM marker (PIP2A-RFP) with ZmHMA2-GFP (Figure 7H) and

323 ZmHMA3-GFP (Figure 7I), respectively. Confocal microscopy analysis of co-infiltrated cells

324 indicated that GFP green signals of ZmHMA2-GFP (Figure 7K) and ZmHMA3-GFP (Figure 7L)

325 overlapped with the RFP signals of the PM marker. These results demonstrated that ZmHMA3

326 and ZmHMA3 proteins were primarily localized on the PM.

327 Discussion

328 The $\mathrm{P}_{1 \mathrm{~B}}$-type ATPases, known as HMAs, play an important role in metal transport in plants. In

329 recent studies, many HMA genes have been identified and researched in Arabidopsis and rice

330 (Jin et al., 2015; Takahashi et al., 2012a). However, a thorough analysis of HMA gene family in

331 maize are still relatively few. In this study, we conducted a comprehensive analysis of the

332 ZmHMA gene family, including identification of members, phylogenetic relationships,

333 expression profiles in different tissues and under $\mathrm{Cd}$ stress conditions, and candidate gene

334 associations with leaf $\mathrm{Cd}$ concentration. The results will assist in understanding the roles of these

$335 \mathrm{ZmHMA2}$ and $\mathrm{ZmHMA3}$ genes and their potential molecular mechanisms in response to Cd

336 stress.

337 A total of 12 ZmHMA genes were identified in the maize genome using sequence

338 comparison and phylogenetic analysis, the number of which was similar in Arabidopsis

339 (AtHMA1 to AtHMA8) and rice (OsHMA1-OsHMA9) (Jin et al., 2015) (Figure 1). The gene

Peer) reviewing PDF | (2019:04:37193:2:0:NEW 30 Aug 2019) 
340 structure analysis of ZmHMA proteins indicated that the $H M A$ genes were highly conserved over

341 the course of evolution since the pericentromeric regions of chromosomes have reduced

342 recombination rates. In addition, most of HMA family members have one or more highly

343 conserved N-terminal and/or C-terminal metal binding sits (MBDs) (Williams \& Mills 2005).

344 Although ZmHMA1-ZmHMA12 shared the E1-E2 ATPase domain, the diversity of motif

345

346

347

348

349

350

351

352

353

354

355

356

357

358

359

360

361

362

363

364

365

366

367

368

369

370

371

372

373 compositions was still large with the number ranging from 2 to 10 motifs. Common motifs 8 and 10 that were characteristic of heavy-metal-associated domain were detected in the N-terminus and were included in the transmembrane (Figure 3B). These variations in motif compositions indicated that specific conserved motifs in the HMA proteins underwent functional divergence to transport different metals. Plant HMAs have a diverse C-terminal region, such as His-rich $(\mathrm{HXH}$, $\mathrm{HH}$ ) and Cys-Cys dipeptides (CC, CXC) (Eren et al., 2006; Williams \& Mills 2005). The Cterminal domain play regulatory roles, such as acting as a metal sensor to regulate the activity of the pump or to interact with pump regulatory proteins (Mills et al., 2010). In the present study, ZmHMA2 protein has a long C-terminal extension of 392 amino acids with 5 cysteine pairs and 16 histidine repeats $(\mathrm{HH}, \mathrm{HXH})$ towards the C-terminus. ZmHMA3 protein has a much shorter C-terminal region (156 amino acids), with 6 cysteine pairs and no histidine stretch (Supplementary Figure S1). The different types of MBDs and their positions in the plant $\mathrm{P}_{1 \mathrm{~B}^{-}}$ ATPases indicated ZmHMAs had various protein functions for metal mobilization and transportation.

Analysis of the temporal and spatial expression patterns of ZmHMA genes may provide useful information for establishing their putative functions. Therefore, we identified tissuespecific expression of $Z m H M A$ genes under a normal growth condition. Our expression result showed that ZmHMA2, ZmHMA6, ZmHMA7, ZmHMA11 and ZmHMA12 were highly and constitutively expressed in the developing tissues, suggesting that these genes should be involved in maintaining the essential metal element during the developmental process (Figure 3A). For instance, OsHMA9 were closely related to ZmHMA11 and function in $\mathrm{Cu}$ mobilization from mature leaves to young leaves (Lee et al., 2007). However, only three genes (ZmHMA3, ZmHMA4 and ZmHMA9) exhibited tissue-specific expression in roots, nodes or anthers.

After being induced by Cd, most of the HMA genes in shoots and leaves (ZmHMA1, ZmHMA5, ZmHMA8, ZmHMA9, ZmHMA11,) were downregulated, whereas only the expression of $\mathrm{ZmHMA3}$ and $\mathrm{ZmHMA5}$ were upregulated (Figure 3B). Based on genetic and functional studies of the $\mathrm{P}_{1 \mathrm{~B}}$-ATPases, these transporters were found in a wide range of organisms and were divided into two groups: copper $(\mathrm{Cu}) /$ silver $(\mathrm{Ag})$ and $\mathrm{Zn} / \mathrm{Co} / \mathrm{Cd} / \mathrm{Pb}$ transporters (Takahashi et al., 2012a). For the $\mathrm{Zn} / \mathrm{Co} / \mathrm{Cd} / \mathrm{Pb}$ subgroup in maize ZmHMA1-ZmHMA4), ZmHMA2 was 
374 more closely related to OsHMA2 in rice. OsHMA2 plays an important role in preferentially

375 distributing $\mathrm{Zn}$ and $\mathrm{Cd}$ and participating in $\mathrm{Zn}$ and $\mathrm{Cd}$ transport to developing seeds in rice

376 (Takahashi et al., 2012b). ZmHMA3 and ZmHMA4 are the homolog of OsHMA3 and may

377 control the $\mathrm{Cd}$ translocation. As previously reported, OsHMA3 transports only $\mathrm{Cd}$ and limits $\mathrm{Cd}$

378 accumulation in the grains (Ueno et al., 2010). However, ZmHMA1 and ZmHMA4 were not

379 expressed or exhibited at extremely low level in different tissues (Figure 3A). Notably, the

380 expression of $Z m H M A 2$ and $Z m H M A 3$ were clearly inducible in the both the shoots and leaves

381 by Cd stress, indicating a possible role for these two candidate genes in regulating $\mathrm{Cd}$

382 transportation (Figure 3B).

383 Supporting this premise is the fact that in the association analysis, the genetic variation of

$384 Z m H M A 2$ and ZmHMA3 was found to be linked to the Cd accumulation variation. After

385 controlling for population structure and relative kinship, one InDel (InDel S1174) in the intron

386 from ZmHMA2 was significantly associated with LCd15-Low and LCd16-Low at seedling stage

$387(P<0.01$, Table 3). This result suggested that ZmHMA2 might play an important role in Cd

388 accumulation under low $\mathrm{Cd}$ condition in maize seedlings. ZmHMA2 was more closely related to

389 the OsHMA2 orthologue in rice (Figure 1). Our findings were similar to that reported by Yamaji

390 et al (Yamaji et al., 2013), showing that OsHMA2 were involved in metal transport at the

391 vegetative growth stage. There was no significant decrease in the leaf $\mathrm{Cd}$ concentration in

392 OsHMA2 mutated plants at the reproductive stage. OsHMA2 was reported to be localized on the

393 plasma membrane (Takahashi et al., 2012b). In the present study, the subcellular localization by

394 transient expression in tobacco demonstrated that ZmHMA2 was also located on the plasma

395 membrane (Figure 6A2-D2). Our results suggested that ZmHMA2 had a similar function with

396 OsHMA2 and plays a role in Cd translation at the vegetative growth stage.

397 In the ZmHMA3, 13 non-synonymous and 7 synonymous loci were significantly associated

398 with leaf $\mathrm{Cd}$ concentration at seedling and at mature stages in 2016 (Table 3). Of these

399 associated loci, 4 loci located in the CDS region and close C-terminus were significantly co-

400 associated with LCd16-Field and LCd15-Field. Further genetic analysis revealed that the coding

401 in the accession with InDel S3517 would be terminated in advance and the polymorphic loci had

402 a greater effect on phenotypic variation for the field environment at the mature stage. The

403 ZmHMA3 seems to play an important role in the control of $\mathrm{Cd}$ accumulation at the maturity stage,

404 not during the vegetative growth stage. Based on phylogenetic analysis, ZmHMA3 shows 72\%

405 identity with OsHMA3. Previous studies have shown that OsHMA3 is localized on the tonoplast

406 of rice root cells to sequestrate $\mathrm{Cd}$ into the vacuoles in the roots, keeping $\mathrm{Cd}$ away from the

407 above-ground tissues and limiting Cd accumulation in the grain (Ueno et al., 2010). In addition, 
408 a variant in C-terminal region perform regulatory roles in OsHMA3 activity (Kumagai et al.,

409 2014). All these results indicated that the variants on the C-terminal region of ZmHMA3 played

410 an important role in controlling $\mathrm{Cd}$ accumulation of maize during the entire growth period. In our

411 study, the subcellular localization demonstrated that ZmHMA3 was located on plasma

412 membrane, which was different from OsHMA3 which was located on the tonoplast. This

413 difference might be explained by the special biological function of HMA3. However, these

414 special biological processes and favorable polymorphisms loci need to be further examined in

415 maize.

\section{Conclusions}

417 The comprehensive analysis of ZmHMA genes would provide overall new insights into their

418 potential involvement in heavy metal transport. In total, 12 members of ZmHMA gene family

419 were identified in maize genome, which were classified into six clusters according to the

420 structural and functional properties. Identified $Z m H M A 2$ and $Z m H M A 3$ genes had a relatively

421 high expression in leaf tissue and as candidate genes to control $\mathrm{Cd}$ accumulation in leaves of

422 maize under various $\mathrm{Cd}$ conditions at seedling and mature stages. More importantly, a significant

423 association in the $\mathrm{ZmHMA3}$ gene with leaf $\mathrm{Cd}$ concentration was detected in that the DNA

424 polymorphisms in the gene CDS region and close C-terminus. The variants on the C-terminal

425 region of ZmHMA3 may in turn contribute to the $\mathrm{Cd}$ transport development across the plasma

426 membrane. Moreover, favorable markers were derived from polymorphic loci within ZmHMA3

427 will represent a valuable genetic resource for limiting $\mathrm{Cd}$ accumulation in maize by marker-

428 assisted breeding. Future studies can be performed for gene validation for the mechanism of $\mathrm{Cd}$

429 accumulation in maize.

\section{Additional Information and Declarations}

\section{Funding}

432 This work was supported by National Key Technologies Research and Development Program

433 (No. 2018YFD0200707, 2018YFD0800600), Education Department of Sichan Province (No.

434 035Z2240), Public Welfare Industry Research of Fujian Province (No. 2017R1026-1).

\section{Competing Interests}

436 The authors declare that they have no competing interests.

\section{Author Contributions}

438 - Yanhua Cao conceived and designed the experiments, performed the experiments, contributed 439 reagents/materials/analysis tools, prepared figures and/or tables, and approved the final draft.

440 - Xiongwei Zhao designed and performed the experiments, analyzed the phenotype data, 
441 approved the final draft.

442 - Yajian Liu performed the experiments, prepared figures and tables, approved the final draft.

443 - Wenmei Wen performed the experiments, prepared figures and/or tables, approved the final

444 draft.

445 - Yalong Wang performed the experiments, prepared figures and/or tables, approved the final 446 draft.

447 - Yiwei Jiang reviewed drafts of the paper, approved the final draft.

448 - Changjian Liao contributed reagents/materials/analysis tools.

449 - Xiaoxun Xu contributed reagents/materials/analysis tools.

450 - Shibing Gao provided pedigree information and experiment materials, approved the final draft.

451 - Yaou Shen contributed reagents/materials/analysis tools.

452 - Hai Lan provided pedigree information and experiment materials, approved the final draft.

453 - Guangtang Pan conceived and designed the experiments, contributed reagents and experiment 454 materials, authored or reviewed drafts of the paper, and approved the final draft.

455 - Haijian Lin conceived and designed the experiments, authored or reviewed drafts of the paper,

456 contributed reagents/materials/analysis tools, and approved the final draft.

457 Data Availability

458 The following information was supplied regarding data availability:

459 The supplemental data is available in Tables S1-S8, and Figure S1 and S2.

460 Supplementary Information

461 Figure S1: Conserved motifs identified from the $H M A$ genes in maize.

462 Figure S2: Alignment of HMA3-like proteins in Maize (ZmHMA2, ZmHMA3 and ZmHMA4),

463 Arabidopsis thaliana (AtHMA2, AtHMA3 and AtHMA4) and rice (OsHMA2, OsHMA3).

464 Table S1: Expression level of ZmHMAs in different tissues and stages of maize plants growing 465 under non-limiting growth conditions.

466 Table S2: List of gene primers used for qRT-PCR assay, sequencing analyses and constructed

467 vectors in the present study.

468 Table S3: Pedigree information for the 103 maize inbred lines in the present study.

469 Table S4: Functional annotation of ZmHMA genes against gene ontology terms.

470 Table S5: Prediction of transmembrane helices in ZmHMA2, ZmHMA3 and ZmHMA4.

471 Table S6: Nucleotide diversity analysis of ZmHMA2 in 103 maize inbred lines.

472 Table S7: Nucleotide diversity analysis of ZmHMA3 in 103 maize inbred lines.

473 Table S8: Prediction analysis of subcellular localization in ZmHMA2 and ZmHMA3 protein

474 using ProtComp.

475 References 
476

477

478

479

480

481

482

483

484

485

486

487

488

489

490

491

492

493

494

495

496

497

498

499

500

501

502

503

504

505

506

507

508

509

Bailey TL, Boden M, Buske FA, Frith M, Grant CE, Clementi L, Ren J, Li WW, and Noble WS. 2009. MEME SUITE: tools for motif discovery and searching. Nucleic Acids Research 37:W202-W208 doi: 10.1093/nar/gkp335

Barrett JC, Fry B, Maller J, and Daly MJ. 2004. Haploview: analysis and visualization of LD and haplotype maps. Bioinformatics 21:263-265 doi: 10.1093/bioinformatics/bth457

Bradbury PJ, Zhang Z, Kroon DE, Casstevens TM, Ramdoss Y, and Buckler ES. 2007. TASSEL: software for association mapping of complex traits in diverse samples. Bioinformatics 23:2633-2635 doi: 10.1093/bioinformatics/btm308

Edgar RC. 2004. MUSCLE: multiple sequence alignment with high accuracy and high throughput. Nucleic Acids Research 32:1792-1797 doi: 10.1093/nar/gkh340

Eren E, Kennedy DC, Maroney MJ, and Argüello JM. 2006. A novel regulatory metal binding domain is present in the $\mathrm{C}$ terminus of Arabidopsis $\mathrm{Zn}^{2+}$-ATPase HMA2. Journal of Biological Chemistry 281:33881-33891 doi: 10.1074/jbc.M605218200

Hu B, Jin J, Guo A, Zhang H, Luo J, and Gao G. 2014. GSDS 2.0: an upgraded gene feature visualization server. Bioinformatics 31:1296-1297 doi: 10.1093/bioinformatics/btu817

Huang Y, Ying H, and Yunxia L. 2009. Combined toxicity of copper and cadmium to six rice genotypes (Oryza sativa L.). Journal of Environmental Sciences 21:647-653 doi: 10.1016/S1001-0742(08)62320-7

Jin T, Chen J, Zhu L, Zhao Y, Guo J, and Huang Y. 2015. Comparative mapping combined with homology-based cloning of the rice genome reveals candidate genes for grain zinc and iron concentration in maize. BMC genomics 16:17 doi: 10.1186/s12863015-0176-1

Kumagai S, Suzuki T, Tezuka K, Satoh-Nagasawa N, Takahashi H, Sakurai K, Watanabe A, Fujimura T, and Akagi H. 2014. Functional analysis of the C-terminal region of the vacuolar cadmium-transporting rice OsHMA3. FEBS letters 588:789-794 doi: 10.1016/j.febslet.2014.01.037

Larkin MA, Blackshields G, Brown N, Chenna R, McGettigan PA, McWilliam H, Valentin F, Wallace IM, Wilm A, and Lopez R. 2007. Clustal W and Clustal X version 2.0. Bioinformatics 23:2947-2948 doi: 10.1093/bioinformatics/btm404

Lee S, Kim Y, Lee Y, and An G. 2007. Rice $\mathrm{P}_{1 \mathrm{~B}}$-type heavy-metal ATPase, OsHMA9, is a metal efflux protein. Plant Physiology 145:831-842 doi: 10.1104/pp.107.102236

Li Z, Ma Z, van der Kuijp TJ, Yuan Z, and Huang L. 2014a. A review of soil heavy metal pollution from mines in China: pollution and health risk assessment. Science of the Total 
Environment 468:843-853 doi: 10.1016/j.scitotenv.2013.08.090

511

512

513

514

515

516

517

518

519

520

521

522

523

524

525

526

527

528

529

530

531

532

533

534

535

536

537

538

539

540

541

542

543

Li Z, Ma Z, van der Kuijp TJ, Yuan Z, and Huang L. 2014b. A review of soil heavy metal pollution from mines in China: pollution and health risk assessment. Science of the Total Environment 468:843-853

Liu L, Du Y, Shen X, Li M, Sun W, Huang J, Liu Z, Tao Y, Zheng Y, and Yan J. 2015. KRN4 controls quantitative variation in maize kernel row number. PLOS Gene 11:e1005670 doi: 10.1371/journal.pgen.1005670

Liu L, Zhang Y, Tang S, Zhao Q, Zhang Z, Zhang H, Dong L, Guo H, and Xie Q. 2010. An efficient system to detect protein ubiquitination by agroinfiltration in Nicotiana benthamiana. Plant Journal 61:893-903 doi: 10.1111/j.1365-313X.2009.04109.x

Mills RF, Valdes B, Duke M, Peaston KA, Lahner B, Salt DE, and Williams LE. 2010. Functional significance of AtHMA4 C-terminal domain in planta. PLoS One 5:e13388 doi: 10.1371/journal.pone.0013388

Morel M, Crouzet J, Gravot A, Auroy P, Leonhardt N, Vavasseur A, and Richaud P. 2009. AtHMA3, a P1B-ATPase allowing $\mathrm{Cd} / \mathrm{Zn} / \mathrm{Co} / \mathrm{Pb}$ vacuolar storage in Arabidopsis. Plant Physiology 149:894-904 doi: 10.1104/pp.108.130294

Rozas J, Ferrer-Mata A, Sánchez-DelBarrio JC, Guirao-Rico S, Librado P, RamosOnsins SE, and Sánchez-Gracia A. 2017. DnaSP 6: DNA Sequence Polymorphism Analysis of Large Data Sets. Molecular Biology and Evolution 34:3299-3302 doi: 10.1093/molbev/msx248

Satoh-Nagasawa N, Mori M, Nakazawa N, Kawamoto T, Nagato Y, Sakurai K, Takahashi H, Watanabe A, and Akagi H. 2011. Mutations in rice (Oryza sativa L.) heavy metal ATPase 2 (OsHMA2) restrict the translocation of zinc and cadmium. Plant \& Cell Physiology 53:213-224 doi: 10.1093/pcp/pcr166

Schefe JH, Lehmann KE, Buschmann IR, Unger T, and Funke-Kaiser H. 2006. Quantitative real-time RT-PCR data analysis: current concepts and the novel "gene expression's CT difference" formula. Journal of molecular medicine 84:901-910 doi: 10.1007/s00109-006-0097-6

Stelpflug SC, Sekhon RS, Vaillancourt B, Hirsch CN, Buell CR, de Leon N, and Kaeppler SM. 2016. An expanded maize gene expression atlas based on RNA sequencing and its use to explore root development. Plant Genome 9:1:16 doi: 10.3835/plantgenome2015.04.0025

Takahashi R, Bashir K, Ishimaru Y, Nishizawa NK, and Nakanishi H. 2012a. The role of heavy-metal ATPases, HMAs, in zinc and cadmium transport in rice. Plant Signal Behav 
544

545

546

547

548

549

550

551

552

553

554

555

556

557

558

559

560

561

562

563

564

565

566

567

568

569

\title{
7:1605-1607 doi: 10.4161/psb.22454
}

\author{
Takahashi R, Ishimaru Y, Shimo H, Ogo Y, Senoura T, Nishizawa NK, and Nakanishi H.
} 2012b. The OsHMA2 transporter is involved in root-to-shoot translocation of $\mathrm{Zn}$ and $\mathrm{Cd}$ in rice. Plant Cell Environ 35:1948-1957 doi: 10.1111/j.1365-3040.2012.02527.x

Ueno D, Yamaji N, Kono I, Huang CF, Ando T, Yano M, and Ma JF. 2010. Gene limiting cadmium accumulation in rice. Proceedings of the National Academy of Sciences of the United States of America 107:16500-16505 doi: 10.1073/pnas.1005396107

Verret F, Gravot A, Auroy P, Leonhardt N, David P, Nussaume L, Vavasseur A, and Richaud P. 2004. Overexpression of AtHMA4 enhances root-to-shoot translocation of zinc and cadmium and plant metal tolerance. FEBS letters 576:306-312 doi: 10.1016/j.febslet.2004.09.023

Williams LE, and Mills RF. 2005. $\mathrm{P}_{1 \mathrm{~B}}$-ATPases-an ancient family of transition metal pumps with diverse functions in plants. Trends in Plant Science 10:491-502 doi: 10.1016/j.tplants.2005.08.008

Yamaji N, Xia XJ, Mitani-Ueno N, Yokosho K, and Ma JF. 2013. Preferential delivery of $\mathrm{Zn}$ to developing tissues in rice is mediated by a P-type ATPases, OsHMA2. Plant Physiology 162:927-939 doi: 10.1104/pp.113.216564

Zhang X, Zhang H, Li L, Lan H, Ren Z, Liu D, Wu L, Liu H, Jaqueth J, and Li B. 2016. Characterizing the population structure and genetic diversity of maize breeding germplasm in Southwest China using genome-wide SNP markers. BMC genomics 17:697 doi: 10.1186/s12864-016-3041-3

Zhao X, Luo L, Cao Y, Liu Y, Li Y, Wu W, Lan Y, Jiang Y, Gao S, and Zhang Z. 2018. Genome-wide association analysis and QTL mapping reveal the genetic control of cadmium accumulation in maize leaf. BMC genomics 19:91 doi: 10.1186/s12864-0174395-x

Peer) reviewing PDF | (2019:04:37193:2:0:NEW 30 Aug 2019) 


\section{Table 1 (on next page)}

List of annotated HMA genes in maize. 


\begin{tabular}{|c|c|c|c|c|c|c|c|c|c|c|}
\hline Gene name & Primary transcript name & Chr & Bin & Genome location & $\begin{array}{l}\text { Gene Size } \\
\text { (bp) }\end{array}$ & $\begin{array}{l}\text { CDS } \\
\text { Size (bp) }\end{array}$ & Exons & $\begin{array}{l}\text { Length } \\
\text { (aa) }\end{array}$ & $\begin{array}{l}\text { Molecular } \\
\text { weight (kDa) }\end{array}$ & $\begin{array}{l}\text { Theoretical } \\
\text { isoelectric point (pI) }\end{array}$ \\
\hline ZmHMA1 & GRMZM2G067853_T02 & 5 & 5.03 & $52,146,625-52,168,252$ & 21627 & 2472 & 13 & 823 & 87.86 & 6.67 \\
\hline ZmHMA2 & GRMZM2G099191_T01 & 5 & 5.03 & $56,451,481-56,458,107$ & 6626 & 3300 & 10 & 1099 & 117.86 & 6.30 \\
\hline ZmHMA3 & GRMZM2G175576_T02 & 2 & 2.06 & $159,040,587-159,044,553$ & 3966 & 2697 & 5 & 898 & 92.56 & 6.67 \\
\hline ZmHMA4 & GRMZM2G455491_T01 & 2 & 2.06 & $159,019,693-159,023,011$ & 3318 & 2895 & 5 & 964 & 96.02 & 7.84 \\
\hline ZmHMA5 & GRMZM2G144083_T01 & 2 & 2.04 & $19,244,753-19,250,983$ & 6230 & 2556 & 6 & 851 & 112.43 & 6.34 \\
\hline ZmHMA6 & GRMZM2G315931_T01 & 4 & 4.08 & $197,229,916-197,239,315$ & 9399 & 2787 & 17 & 928 & 97.36 & 7.10 \\
\hline ZmHMA7 & GRMZM2G029951_T01 & 5 & 5.04 & $121,445,070-121,449,579$ & 4509 & 2943 & 10 & 980 & 106.28 & 5.33 \\
\hline$Z m H M A 8$ & GRMZM5G855347_T01 & 1 & 1.03 & $16,331,867-16,338,054$ & 6187 & 2640 & 16 & 879 & 101.48 & 6.61 \\
\hline ZmHMA9 & GRMZM2G404702_T01 & 9 & 9.04 & $109,908,973-109,914,816$ & 5843 & 3072 & 10 & 1023 & 109.52 & 5.31 \\
\hline ZmHMA10 & GRMZM2G143512_T01 & 2 & 2.04 & $19,329,257-19,337,335$ & 8078 & 3000 & 6 & 999 & 102.37 & 6.46 \\
\hline ZmHMA11 & GRMZM2G010152_T01 & 4 & 4.10 & $236,277,989-236,283,316$ & 5327 & 2997 & 9 & 998 & 107.58 & 5.33 \\
\hline ZmHMA12 & GRMZM2G151406_T01 & 9 & 9.04 & $109,906,735-109,908,497$ & 1761 & 1200 & 4 & 400 & 42.3 & 5.6 \\
\hline
\end{tabular}




\section{Table 2 (on next page)}

Nucleotide diversity and neutrality test of $Z m H M A 2$ and ZmHMA3. 


\begin{tabular}{|c|c|c|c|c|c|c|c|c|c|c|}
\hline Gene & Regions & $\begin{array}{c}\text { Length } \\
\text { (bp) }\end{array}$ & InDel & SNP & $\begin{array}{c}\text { Haplotype } \\
\text { number }\end{array}$ & $\begin{array}{c}\pi \\
\left(\times 10^{-3}\right) \\
\end{array}$ & $\begin{array}{c}\theta \omega \\
\left(\times 10^{-3}\right) \\
\end{array}$ & $\begin{array}{c}\text { Tajima' } \\
\text { s } D\end{array}$ & $\begin{array}{c}\text { Fu and } \\
\text { Li's } D \\
\end{array}$ & $\begin{array}{c}\text { Fu and } \\
\text { Li's } F\end{array}$ \\
\hline \multirow{5}{*}{ ZmHMA2 } & 5'-UTR & 322 & 4 & 9 & 6 & 9.38 & 1.54 & 2.01 & 1.26 & $1.73^{*}$ \\
\hline & CDS & 3300 & 5 & 57 & 42 & 6.24 & 22.28 & -0.26 & -1.24 & -0.93 \\
\hline & Intron & 2600 & 50 & 128 & 75 & 17.58 & 43.02 & 0.09 & -0.56 & -0.31 \\
\hline & 3'-UTR & 409 & 3 & 11 & 12 & 10.82 & 4.23 & 0.12 & -1.15 & -0.77 \\
\hline & Overall & 6631 & 62 & 205 & 85 & 11.05 & 71.06 & 0.04 & -0.81 & -0.48 \\
\hline \multirow{5}{*}{$Z m H M A 3$} & 5'-UTR & 187 & 0 & 3 & 7 & 6.26 & 1.34 & -0.30 & 0.31 & 0.12 \\
\hline & CDS & 2923 & 5 & 68 & 39 & 7.36 & 21.51 & -0.12 & -1.48 & -1.02 \\
\hline & Intron & 413 & 9 & 33 & 25 & 33.57 & 8.83 & 1.40 & 0.13 & 0.76 \\
\hline & 3'-UTR & 447 & 10 & 35 & 17 & 18.85 & 8.83 & -0.29 & $1.55^{*}$ & 0.90 \\
\hline & Overall & 3970 & 24 & 139 & 48 & 11.20 & 40.52 & 0.18 & -0.39 & -0.16 \\
\hline
\end{tabular}

1 


\section{Table 3 (on next page)}

Associations between the natural variations within ZmHMA2 and ZmHMA3 genes and leaf $\mathrm{Cd}$ concentration under various $\mathrm{Cd}$ conditions by using kinship $(\mathrm{K})$ and a population structure controlled model.

a The letters indicate nucleotide polymorphisms and the numbers indicate the inserted or deleted nucleotides. Under lined letters and numbers represent the minor alleles. MAF represent minor allele frequency. $\mathrm{R}^{2}$ represent explained phenotype variation. 


\begin{tabular}{|c|c|c|c|c|c|c|c|}
\hline Gene & Trait & Site & Allele $^{\mathrm{a}}$ & MAF & $P\left(\times 10^{-3}\right)$ & $\mathrm{R}^{2}(\%)$ & \multirow[t]{3}{*}{ Amino Acid Variations } \\
\hline \multirow{2}{*}{ ZmHMA2 } & LCd15-Low & \multirow{2}{*}{1174} & \multirow{2}{*}{$3 / \underline{0}$} & \multirow{2}{*}{0.24} & 7.96 & 7.57 & \\
\hline & LCd16-Low & & & & 8.99 & 8.15 & \\
\hline \multirow{30}{*}{ ZmHMA3 } & LCd16-Field & \multirow{2}{*}{720} & \multirow{2}{*}{$\mathrm{C} / \underline{\mathrm{A}}$} & \multirow{2}{*}{0.21} & 6.60 & 8.35 & \multirow{6}{*}{$\operatorname{ALA}(\mathrm{A}) / \mathrm{GLY}(\mathrm{G})$} \\
\hline & LCd15-Middle & & & & 8.47 & 7.66 & \\
\hline & LCd16-Middle & 917 & $\mathrm{C} / \underline{\mathrm{G}}$ & 0.18 & 2.09 & 10.47 & \\
\hline & LCd16-Field & \multirow{2}{*}{921} & \multirow{2}{*}{$\mathrm{C} / \underline{\mathrm{G}}$} & \multirow{2}{*}{0.21} & 6.60 & 8.35 & \\
\hline & LCd15-Middle & & & & 8.47 & 7.66 & \\
\hline & LCd15-Low & 942 & $\mathrm{C} / \underline{\mathrm{G}}$ & 0.47 & 3.32 & 10.64 & \\
\hline & LCd16-Field & 944 & $\mathrm{C} / \underline{\mathrm{G}}$ & 0.27 & 2.48 & 10.47 & \multirow[t]{6}{*}{$\operatorname{ALA}(\mathrm{A}) / \mathrm{GLY}(\mathrm{G})$} \\
\hline & LCd15-Low & 951 & $\mathrm{~A} / \underline{\mathrm{C}}$ & 0.46 & 3.32 & 10.64 & \\
\hline & LCd16-Middle & 957 & $\mathrm{G} / \underline{\mathrm{A}}$ & 0.17 & 2.85 & 9.81 & \\
\hline & LCd15-Low & 1467 & $\underline{\mathrm{C}} / \mathrm{T}$ & 0.70 & 5.48 & 9.46 & \\
\hline & LCd15-Low & 1522 & $\underline{\mathrm{T}} / \mathrm{A}$ & 0.32 & 4.04 & 10.17 & \\
\hline & LCd15-Low & 1526 & $\underline{2} / 0$ & 0.69 & 4.04 & 10.17 & \\
\hline & LCd16-Field & 2540 & $\mathrm{~T} / \underline{\mathrm{A}}$ & 0.09 & 0.48 & 14.17 & \multirow[t]{4}{*}{ SER (S) / THR (T) } \\
\hline & LCd16-Field & 2809 & $\mathrm{C} / \underline{\mathrm{G}}$ & 0.12 & 0.99 & 12.52 & \\
\hline & LCd15-Field & \multirow{2}{*}{3097} & \multirow{2}{*}{$\mathrm{G} / \underline{\mathrm{A}}$} & \multirow{2}{*}{0.09} & 5.44 & 8.33 & \\
\hline & LCd16-Field & & & & 0.21 & 16.15 & \\
\hline & LCd15-Field & \multirow{2}{*}{3114} & \multirow{2}{*}{$\mathrm{G} / \underline{\mathrm{C}}$} & \multirow{2}{*}{0.09} & 5.44 & 8.33 & \multirow{2}{*}{ GLY (G) / ALA (A) } \\
\hline & LCd16-Field & & & & 0.21 & 16.15 & \\
\hline & LCd15-Field & \multirow{2}{*}{3297} & \multirow{2}{*}{$\mathrm{T} / \underline{\mathrm{C}}$} & \multirow{2}{*}{0.09} & 5.44 & 8.33 & \multirow{2}{*}{$\operatorname{VLA}(\mathrm{V}) / \operatorname{ALA}(\mathrm{A})$} \\
\hline & LCd16-Field & & & & 0.21 & 16.15 & \\
\hline & LCd15-Field & \multirow{2}{*}{3517} & \multirow{2}{*}{$9 / \underline{0}$} & \multirow{2}{*}{0.09} & 5.44 & 8.33 & VI $\Delta(\mathrm{V}) /$ Dro $(\mathrm{D}) /$ Ston \\
\hline & LCd16-Field & & & & 0.21 & 16.15 & VLA (V) / PTO (I) / SLP \\
\hline & LCd15-Field & 3504 & $6 / 0$ & 000 & 5.44 & 8.33 & $\Delta \mathrm{I} \Delta(\Delta) / \mathrm{I} \rho \mathrm{ou}(\mathrm{I})$ \\
\hline & LCd16-Field & 3594 & $6 / \underline{0}$ & 0.09 & 0.21 & 16.15 & ALA (A)/ Leu (L) \\
\hline & LCd15-Field & 2602 & $C / 4$ & 000 & 4.97 & 8.76 & \\
\hline & LCd16-Field & 3092 & Cl一 & 0.09 & 0.21 & 16.28 & \\
\hline & LCd15-Field & 3605 & $0 / 0$ & 000 & 5.44 & 8.33 & \\
\hline & LCd16-Field & 3695 & $0 / \underline{9}$ & 0.09 & 0.21 & 16.15 & \\
\hline & LCd15-Field & 2702 & $0 / 2$ & 000 & 5.44 & 8.33 & \\
\hline & LCd16-Field & 3192 & $0 / \underline{\underline{z}}$ & 0.09 & 0.21 & 16.15 & \\
\hline
\end{tabular}

1 
Figure 1

Phylogenetic analysis of HMA proteins between maize (ZmHMA1 to ZmHMA12), rice (OsHMA1 to OsHMA9) and Arabidopsis (AtHMA1 to AtHMA8).

On the basis of genetic and functional studies of the $\mathrm{P}_{1 \mathrm{~B}}$-ATPases, zinc (Zn)/cobalt (Co)/cadmium (Cd)/lead (Pb) group was highlighted in purple, the copper (Cu)/silver ( $\mathrm{Ag})$ group was highlighted in green. The number from I to VI represent different six branches. 


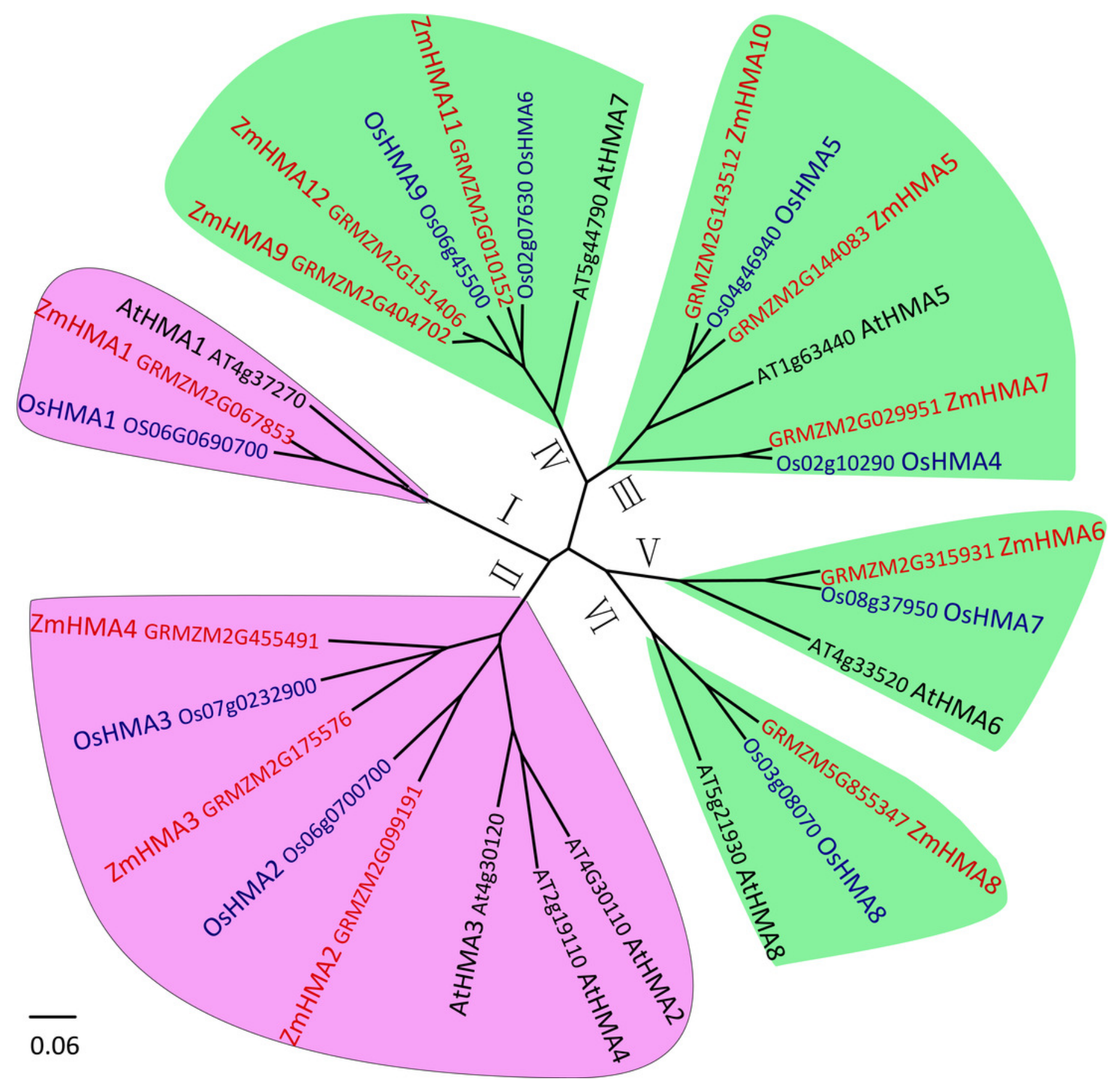


Figure 2

Phylogenetic relationships (A), motif compositions (B) and gene structure (C) within the HMA family in maize.

Different motifs were numbered from 1 to 10 and motifs labeled in the same color indicate that the same conserved domains were present in the different motifs.

A

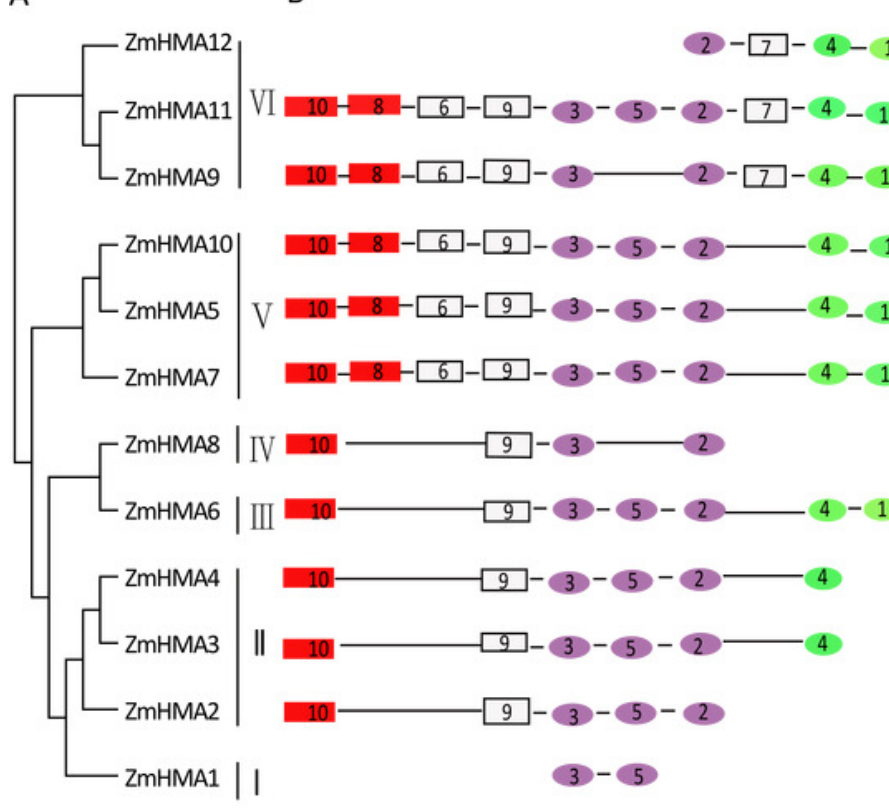

C
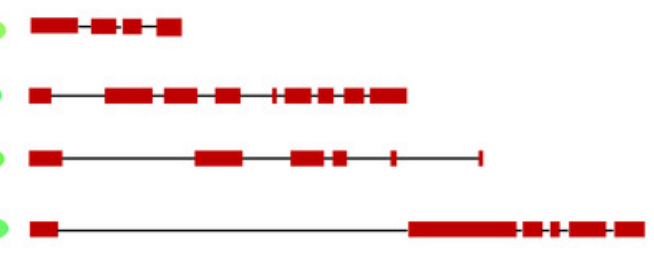

1.

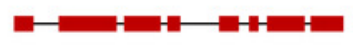

IHe-
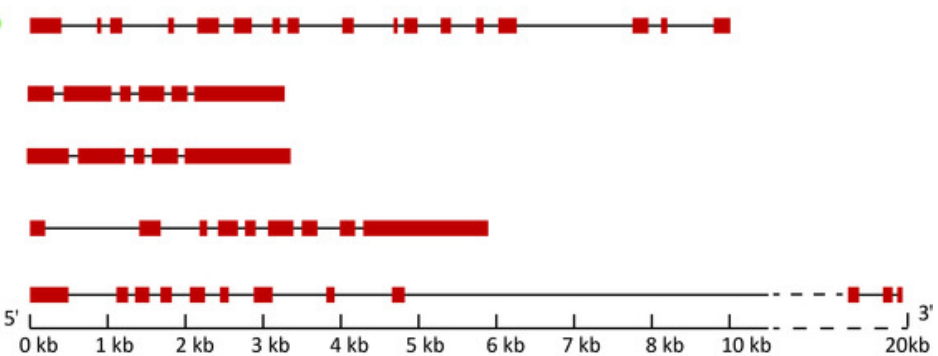


\section{Figure 3}

Expression profiles of eleven ZmHMA genes in maize.

(A) A heat map illustrating levels of gene expression of the 12 ZmHMAs in 13 different tissues from various developmental stages. FPKM values were shown in different colors representing expression levels as indicated by the scale bar. (B-L) Relative expression levels of 11 ZmHMAs in maize B73 root, leaf and stem tissues grown hydroponically under normal (ck) and Cd-additive conditions $(200 \mu \mathrm{M})$ at the vegetative growth stage for $12 \mathrm{~h}, 24 \mathrm{~h}$ and $48 \mathrm{~h}$. The comparisons of gene expression under $\mathrm{ck}$ and $\mathrm{Cd}$ stress conditions were performed using a two-sided t-test: ${ }^{*} p \leq 0.05,{ }^{* *} p \leq 0.01$. Bars indicate standard deviation. 


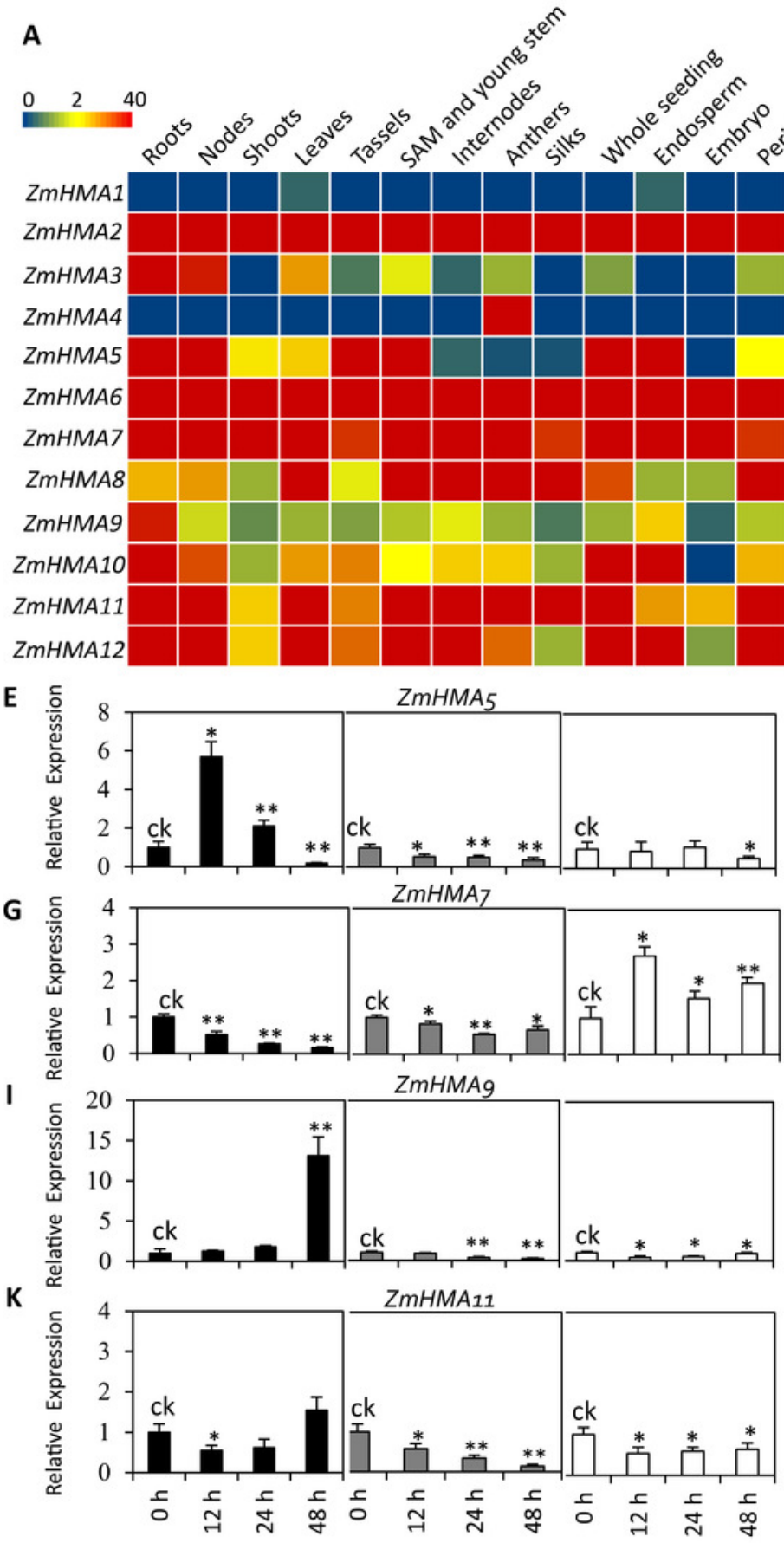

B

$\square$ Root $\square$ Stem $\square$ Leaf

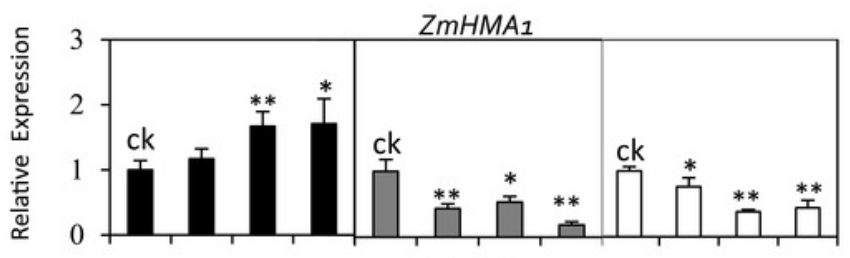

C

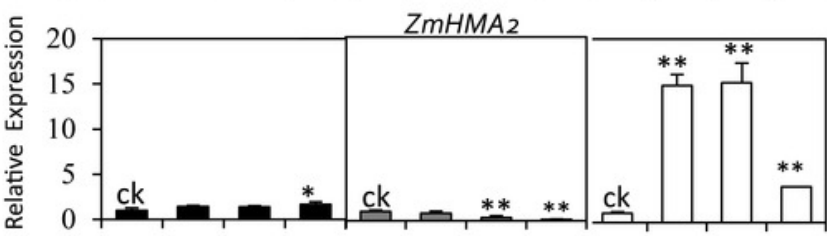

D

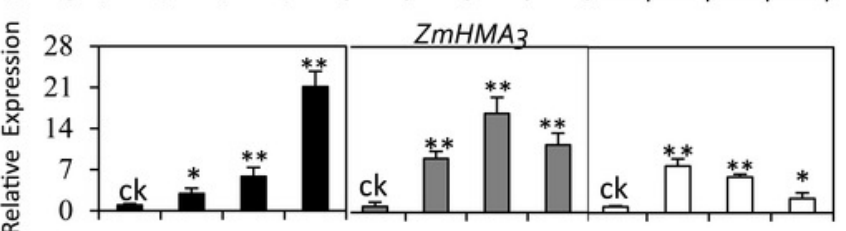

$\mathbf{F}$

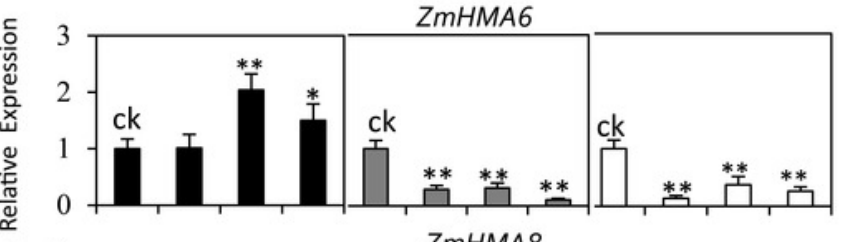

H

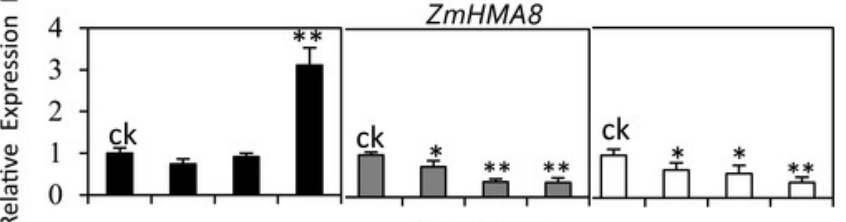

J

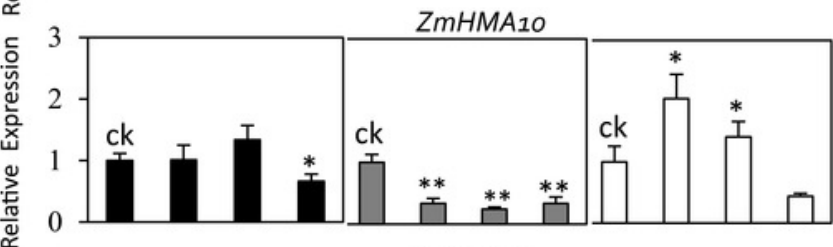

L

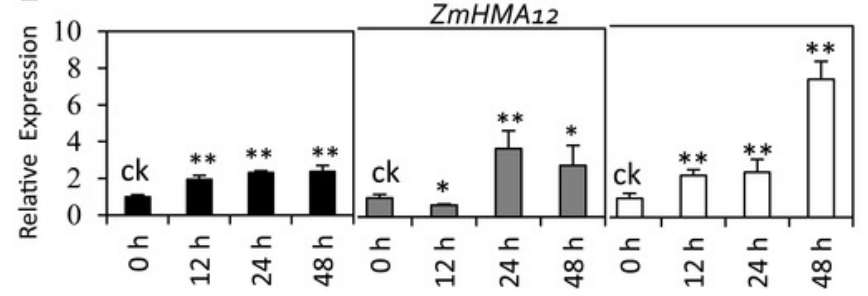


Figure 4

Plot of linkage disequilibrium $\left(r^{2}\right)$ decay against physical distance between SNPs from ZmHMA2 and ZmHMA3, respectively.

Measurement was performed by averaging $r^{2}$ values of allele frequencies over a distance of $100 \mathrm{bp}$ and plotting the values against distance (bp).

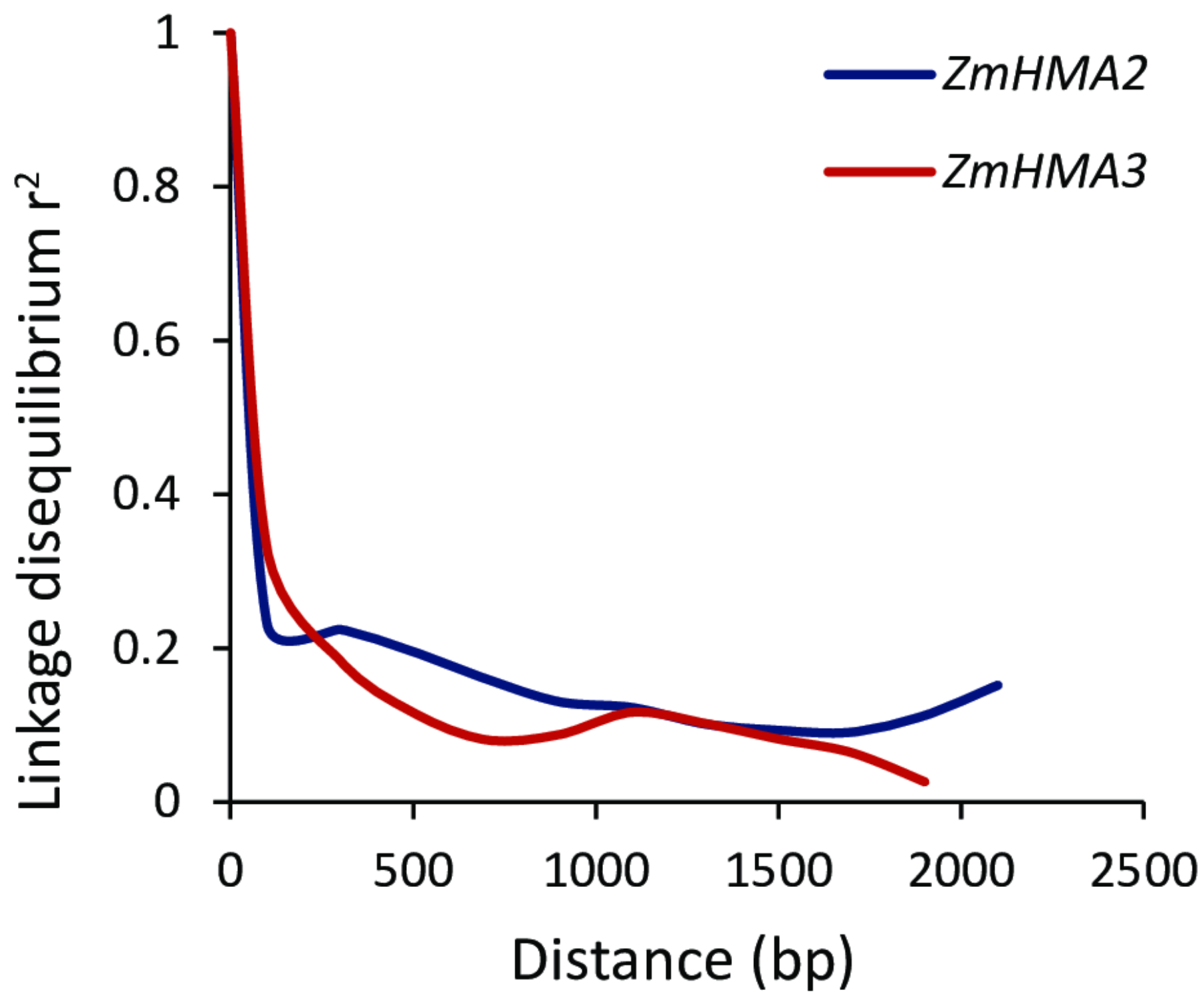




\section{Figure 5}

Natural variation within $\mathrm{ZmHMA3}$ was associated with $\mathrm{Cd}$ concentration in leaves.

(A) Natural variation in ZmHMA3 associated with Cd concentration in leaves under different $C d$ conditions at seeding stage and mature stage. The $P$ value is shown on a - $\log _{10}$ scale. SNP represent single nucleotide polymorphism. InDel represent insertion or deletion. A schematic diagram of the entire gene structure is presented as the $x$-axis, including white and black boxes showing as UTRs and exons, respectively. (B) The pattern of pair wise linkage disequilibrium (LD) of DNA polymorphisms (MAF $>0.05$ ) in decay of ZmHMA3. The level of LD $\left(r^{2}\right)$ values is indicated with color key. 


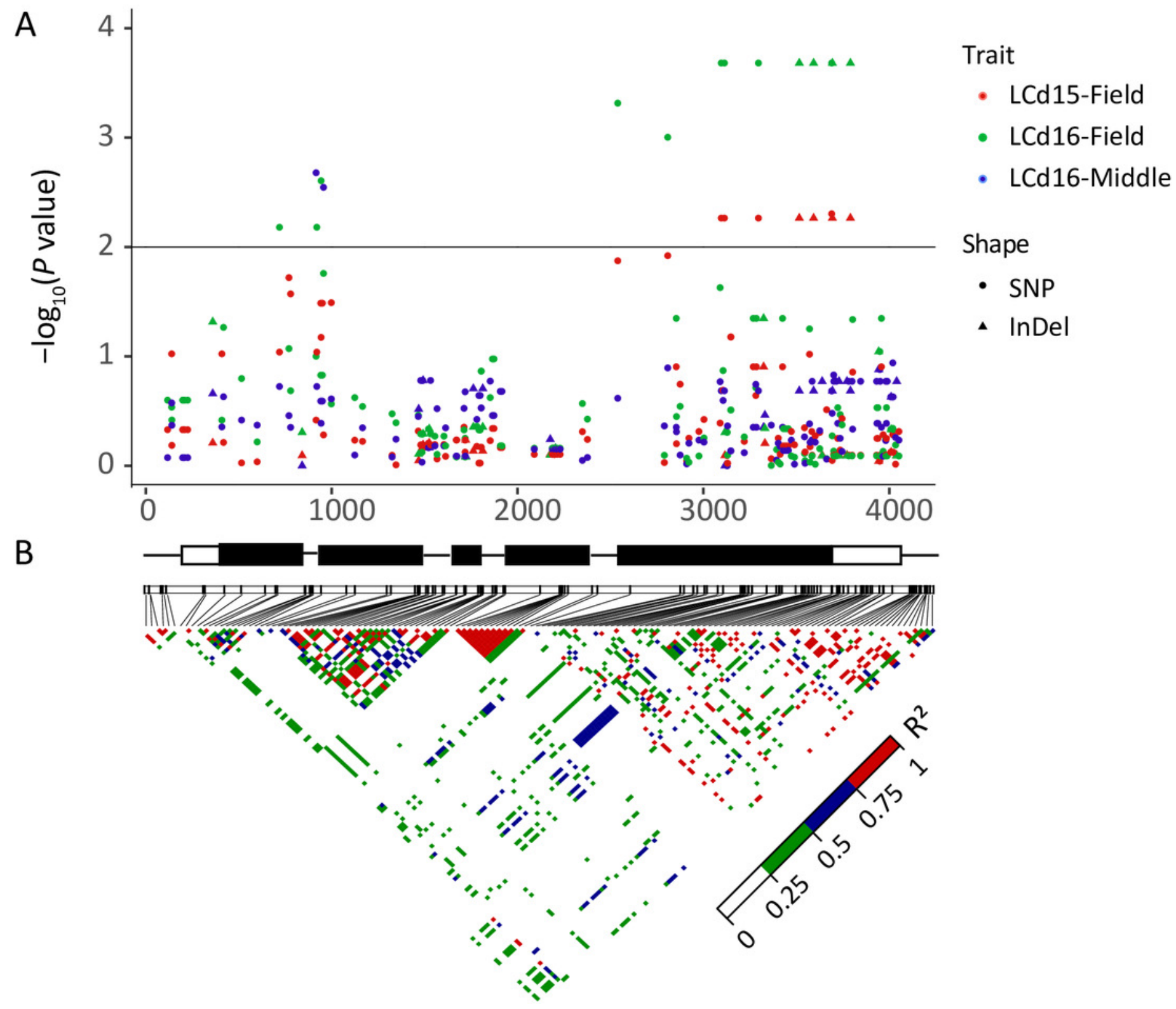




\section{Figure 6}

Genetic effects of $\mathrm{Cd}$ accumulation in leaves were determined by significant SNPs or InDels in ZmHMA2 and ZmHMA3.

The number represents the number of inbred lines homozygous for a determined allelic variant. $\Delta m$, the difference in mean leaf $\mathrm{Cd}$ concentration between minor alleles and major alleles over two years and two replications. Differences between the alleles were analyzed by mixed linear model (MLM, Phenotype $\sim$ SNP + Structure + Kinship). $R^{2}$ values from MLM of the data show the percentage the effect of the allele. (A) Allelic variations of InDel S1174 (3/0) in ZmHMA2 associated with leaf $\mathrm{Cd}$ concentration under low $\mathrm{Cd}$ condition at seeding stage. (B) Allelic variations of S917 (G/C) in ZmHMA3 associated with leaf Cd concentration under high Cd condition at seeding stage. (C) Multi-allelic variations of S3114 (G/C), S3297 (T/C), InDel S3517 (9/0) in ZmHMA3 associated with leaf Cd concentration in the field.

A

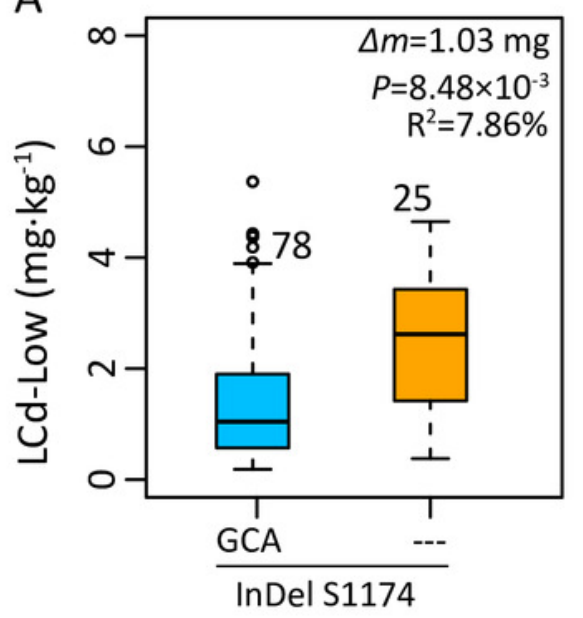

B

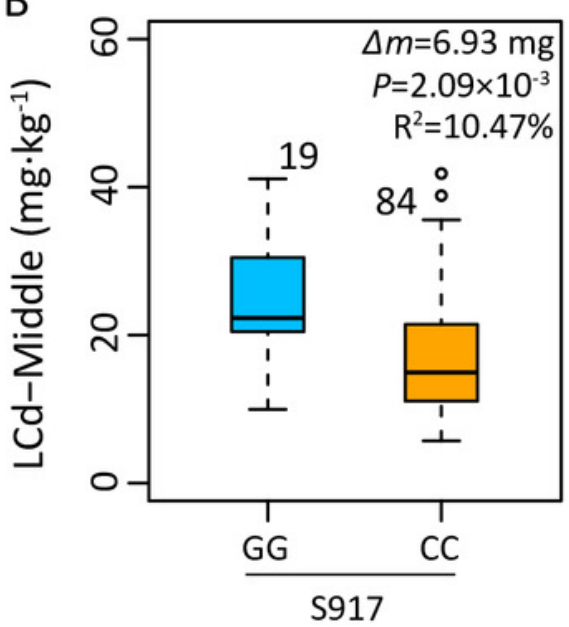

C

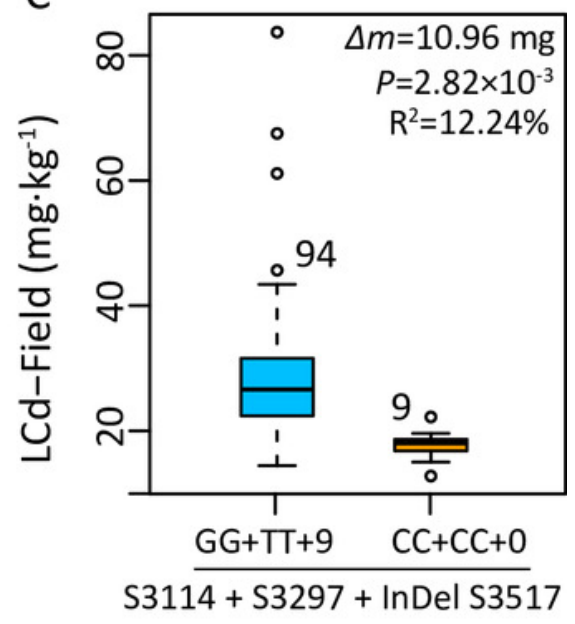




\section{Figure 7}

Subcellular localization of ZmHMA2 and ZmHMA3 in leaves.

(A-C) Tobacco cells under bright-field illumination. (D-F) Location of the fusion proteins, including the empty vector control, ZmHMA2-GFP and ZmHMA3-GFP under GFP-field. (G-I) show co-expression of a plasma marker (PIP2A-RFP), ZmHMA3-GFP and ZmHMA3-GFP under RFP-field . (J-L) Merged bright-field, GFP-field and RFP-field images. Scale Bars, $30 \mathrm{~mm}$.

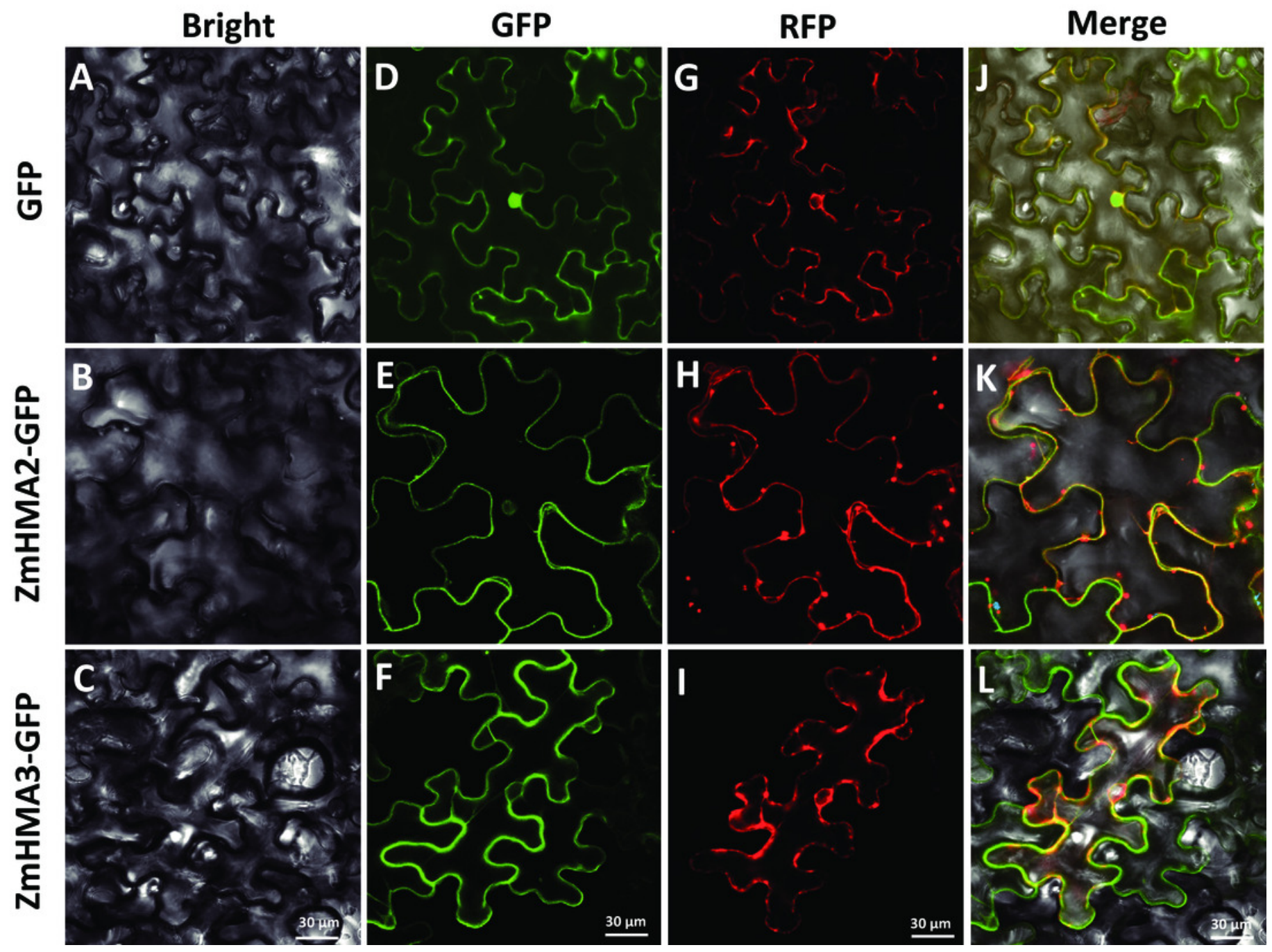

